University of Massachusetts Amherst

ScholarWorks@UMass Amherst

University Libraries Publication Series

University Libraries

January 2007

\title{
Protocol Analysis of a Federated Search Tool: Designing for Users
}

Emily R. Alling

University of Massachusetts - Amherst, ealling@library.umass.edu

Rachael Naismith

Springfield College, rnaismith@spfldcol.edu

Follow this and additional works at: https://scholarworks.umass.edu/librarian_pubs

Part of the Library and Information Science Commons

\section{Recommended Citation}

Alling, Emily R. and Naismith, Rachael, "Protocol Analysis of a Federated Search Tool: Designing for Users" (2007). Internet Reference Services Quarterly. 1.

Retrieved from https://scholarworks.umass.edu/librarian_pubs/1

This Article is brought to you for free and open access by the University Libraries at ScholarWorks@UMass Amherst. It has been accepted for inclusion in University Libraries Publication Series by an authorized administrator of ScholarWorks@UMass Amherst. For more information, please contact scholarworks@library.umass.edu. 


\title{
Protocol analysis of a federated search tool: designing for users
}

\author{
Emily Alling \\ Rachael Naismith
}

Summary. Librarians at Springfield College conducted usability testing of Endeavor's federated search tool, ENCompass for Resource Access. The purpose of the testing was to make informed decisions prior to customizing the look and function of the software's interface in order to make the product more usable for their patrons. Protocol, or think-aloud, analysis was selected as a testing and analysis method. Subjects from the general college community were recruited and given a list of tasks to perform on ENCompass, and they were asked to speak all of their thoughts out loud as they worked. Upon analyzing the test results, researchers found that subjects' problems fell into certain categories, such as unfamiliarity with terms or navigation from screen to screen. The researchers were able to use their findings to recommend extensive revisions to the interface, which improved usability for this library's patrons.

Keywords: protocol analysis, usability testing, federated search

Emily Alling is Coordinator, Learning Commons and Undergraduate Library Services, W.E.B. Du Bois Library, University of Massachusetts, Amherst, 154 Hicks Way, Amherst, MA 01003 (E-mail: ealling @ library.umass.edu). Rachael Naismith is Assistant Director for Information and Research, Babson Library, Springfield College, 263 Alden Street, Springfield, MA 01109 (E-mail: Rachael_Naismith@spfldcol.edu).

Final peer-reviewed version.

[Haworth co-indexing entry note]: "Protocol Analysis of a Federated Search Tool: Designing for Users." Alling, Emily and Rachael Naismith. Co-published simultaneously in Internet Reference Services Quarterly (The Haworth Information Press, an imprint of The Haworth Press, Inc.) Vol. 12, No. 1/2, 2007, pp. 195-210; and: Federated Search: Solution or Setback for Online Library Services (ed: Christopher N. Cox) The Haworth Information Press, an imprint of The Haworth Press, Inc., 2007, pp. 195-210. Single or multiple copies of this article are available for a fee from The Haworth Document Delivery Service [1-800-HAWORTH, 9:00 a.m. - 5:00 p.m. (EST). E-mail address: docdelivery@haworthpress.com].

Available online at http://irsq.haworthpress.com (C) 2007 by The Haworth Press, Inc. All rights reserved. doi:10.1300/J136v12n01_10 


\section{INTRODUCTION}

For years, library professionals and patrons have been forced to adapt their searching behaviors to the interfaces that library systems vendors have shipped with their products. While some products' search interfaces are simple, well-designed, and intuitive, poorly-designed interfaces can create all types of usability nightmares. These can arise from jargon, unclear navigation, poorly placed elements, or features which serve only to distract or annoy.

Increasingly, however, library systems vendors are shipping products whose interfaces can be customized by the libraries that purchase them. As a result, librarians are now in the fortunate, but sometimes daunting, position of deciding whether and how to modify the interfaces of various types of tools in order to best meet the needs of their patrons.

How can library professionals make informed decisions about modifying an interface? Librarians' intuitions about how users search are often directly contradicted by actual users' behaviors and preferences. Thus, gathering data on how users really interact with search tools is crucial when designing or modifying an interface.

Once changes to an interface have been decided upon, libraries face a second question: Who will do the actual work of changing the interface? Many libraries lack staff members who possess the coding or programming skills that are necessary to make these changes. The case for training and hiring librarians who can code is becoming stronger every day, to the point where an entire conference-Code $4 \mathrm{Lib}-$ has been created. However, smaller libraries and those with limited resources are still 
hard-pressed to find or develop local programming expertise. How can these libraries ensure that their users are not left behind?

In 2003, Babson Library (Springfield College, Springfield, MA) purchased ENCompass for Resource Access, a federated search tool developed and marketed by Endeavor Information Systems, Inc. ENCompass, which is based on an XML platform, allows extensive customization. When the library staff examined the "out of the box" version of ENCompass (which was renamed Articles Plus at Springfield College), they immediately identified certain features that they felt would be problematic for users. The library staff wanted to discern whether users would actually have difficulty with these features, how they would handle the problems that arose, and what improvements to the interface could be made to alleviate the problems.

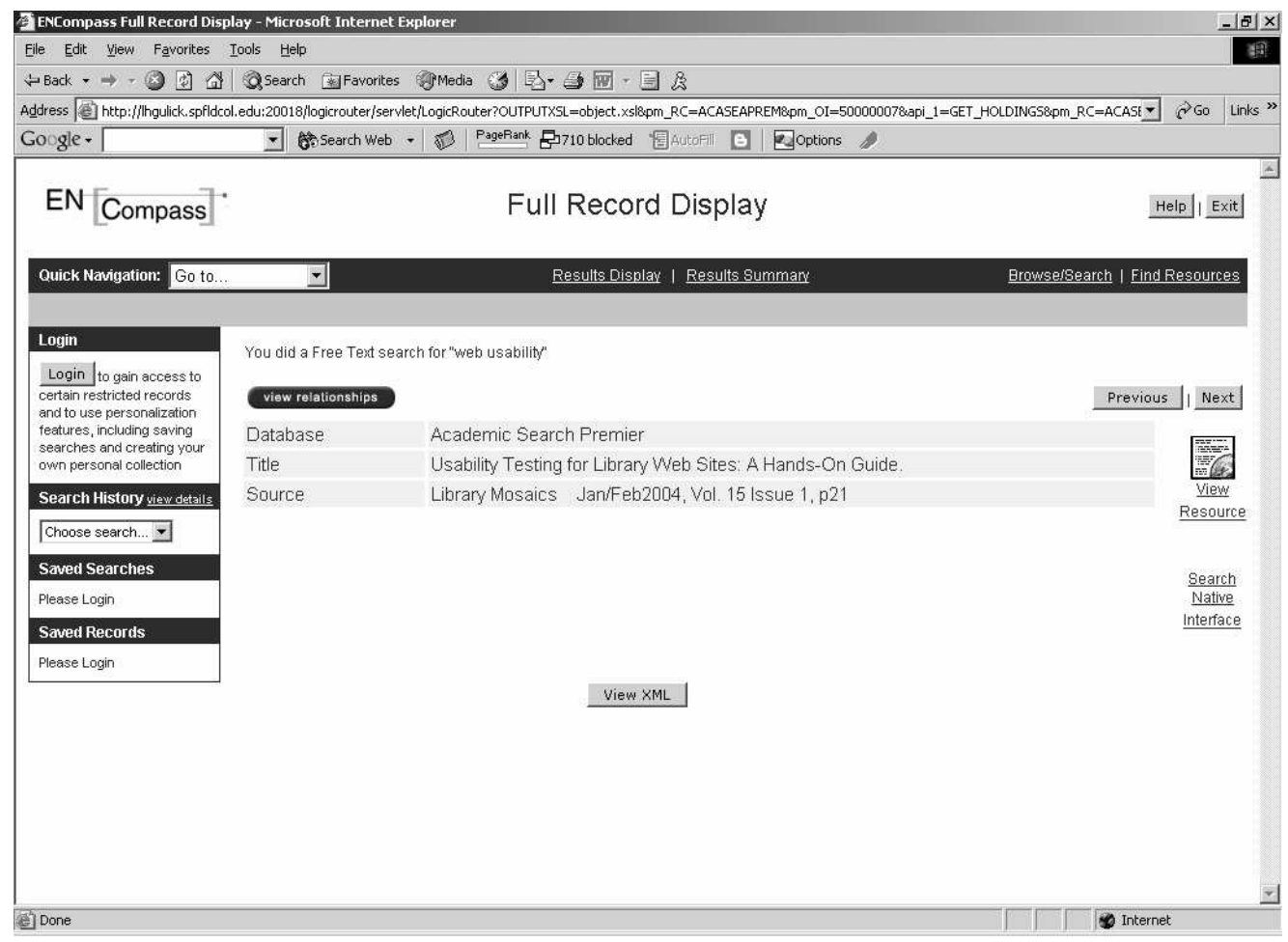

Protocol analysis of federated search

Page 3 of 22 
(Figure 1 -- Out-of-the-box version of ENCompass: display of an individual record.)

In order to make informed decisions about modifying ENCompass, librarians at Springfield College conducted a usability study with a testing technique called protocol analysis, or think-aloud analysis. Protocol analysis was selected as the method of usability testing because it was an excellent way to observe individuals using ENCompass to perform common information-seeking tasks and to get immediate feedback on problems they encountered, as well as their successes.

More significantly, protocol analysis fit the needs of Babson Library, a small academic library with budget limitations. Babson Library has a staff of seventeen, no systems librarian, and no librarians with expertise in usability testing or in federated searching software. Protocol analysis, with its low-technology and low-cost features, provided a means of testing that the Library could afford, making use of existing staff capabilities. For a small college library with time and cost limits, it was an ideal method.

When the time came to implement the recommendations that arose from the protocol analysis of ENCompass, Babson Library drew upon its own staff's limited programming abilities to enact some basic changes, and turned to a supportive community of other ENCompass users, as well as the vendor's user support staff, to accomplish the rest.

\section{Literature review}


Usability studies and techniques are well documented in the library and information science literature. Pace (2003) describes various usability testing techniques, including focus groups, surveys, interviews, and card-sorting exercises. Usability studies have been performed on Web sites (Letnikova 2003 provides an annotated bibliography), online catalogs (Novotny 2004), digital libraries (Van House et al. 1996), and--increasingly--federated search tools (Cervone 2005; Ponsford 2005; Schmidt and Wrubel 2005; Tallent 2004). The studies that have been performed on federated search tools report a number of common findings:

- The concept of federated search remains unfamiliar to many library users; as Cervone points out, "one of the first hurdles to overcome is that the term 'metasearch' means absolutely nothing to most people" (Cervone 2005,11).

- Forcing users to take any steps before typing in their search query, such as choosing databases or categories to be searched, leads to confusion and problems (Cervone 2005,12, Tallent 2004,73).

- Users have a clear mental model of searching that is based on Google. Students enter queries "as if they were searching in a search engine," including using the plus sign operator and assuming the presence of a spell-check feature (Schmidt and Wrubel 2005). They assume an AND between search words and use quotation marks to surround phrases (Ponsford 2005).

- Not surprisingly, then, users prefer a simple interface and tend to overlook or ignore functionality beyond a basic search box. Tallent $(2004,70)$ noted "little interest or knowledge of advanced searching techniques, such as authorized 
subject searching or combining various index searches." Ponsford (2005) reported that, of the subjects tested, "no one saw the advanced search at all."

- Regarding the presentation of results, users seem to prefer and expect relevancy ranking: "in a Google world, the best results are listed first" (Tallent 2004:71). However, many federated search tools display results in reverse chronological order or alphabetically.

- Both when formulating their searches and when evaluating their results, users are concerned with the format of information they are retrieving (books, journal articles, websites, etc.) as well as full text availability (Schmidt and Wrubel 2005; Ponsford 2005).

\section{Protocol analysis}

The technique employed for the usability study described in this article is protocol analysis, also known as think-aloud analysis. Protocol analysis--the use of subjects' verbalized thoughts as data--was first described by K. Anders Ericsson and Herbert A. Simon in a 1980 Psychological Review article. The authors subsequently released a book-length treatment of the topic, Protocol Analysis: Verbal Reports as Data (1993). The main premise of protocol analysis is that having subjects think aloud will "provide researchers with useful information to aid their inferences about how target tasks are normally carried out when thinking aloud is not required" (Gilhooly \& Green 1996, 53).

Some hallmarks of protocol analysis: 
- In protocol analysis, subjects speak their thoughts out loud while performing one or more tasks--the verbal reports are concurrent with the task performance. Other usability techniques, such as focus groups or post-test interviews, may rely on retrospective reports, which have been shown to be less reliable (Gilhooly \& Green 1996, 51).

- Protocol analysis requires comparatively few subjects. Nielsen (1994) demonstrates that testing three to five subjects using think-aloud analysis produces the maximum cost-benefit ratio: "As you add more and more users, you learn less and less because you will keep seeing the same things again and again.... After the fifth user, you are wasting your time by observing the same findings repeatedly but not learning much new."

- The amount of time spent reviewing, analyzing, and coding test results can be substantial. The conventional wisdom is that for each hour of testing, up to ten hours of analysis will be required. In this study, the actual ratio was closer to six hours of analysis per hour of testing. However, the amount of time spent on analysis is balanced by the fact that comparatively few hours of testing are needed in order to harvest most useful data when employing protocol analysis. In this study, four subjects yielded approximately three hours of results for the researchers to analyze.

- During testing, researchers do not intervene in subjects' test performance in any way, other than to remind them to "keep talking" if they fall silent for an extended period. 
- By designing open-ended questions and tasks that engage users in all parts of a system, researchers can create an environment where unanticipated or unexpected problems, interactions, or user preferences may emerge. Wiedenbeck et al. (1989: 26) write: "Because think-aloud protocol analysis is commonly used for domains where things are not well understood, it is normal to have sequences which surprise the people running the study. The strength of using less-structured protocol studies over more controlled experimentation is the opportunity to observe these events which are not anticipated."

- Subjects' activities and speech are recorded for later analysis. A number of recording techniques have been reported in the literature, including videotaping, audio recording, screen capture software such as TechSmith Camtasia or Macromedia Captivate (Mack et al. 2004); and, in the lowest-budget scenario, extensive, close note-taking.

\section{Test design and administration}

The researchers in this study designed seven tasks for subjects to perform during testing (Figure 2). Most of the tasks were designed to test whether certain features and vocabulary in ENCompass would be comprehensible to users. For example, the out-of-the-box version of ENCompass offered an icon labeled "Search Native Interface." Upon clicking this icon, the user would exit federated searching and jump directly into a single database (e.g., PsycINFO, ERIC). The term "Native Interface" is the type of jargon that might stump users, so the researchers made sure that at least one of the tasks assessed whether they understood its meaning. In addition to these 
"hunch questions," researchers included questions that required users to perform common types of searches (known item searches, topical searches, searches for different formats of information) and employ as many of the system's navigational elements and functions as possible (revising a search, moving between results lists and individual records, printing, etc.).

\section{Figure 2. List of tasks performed by subjects during testing}

1) Find information on fitness and children.

2a) Start a new search. Find one book and one article on treatment for drug addiction. 2b) Print one of the records out.

3a) Find some articles about genetically modified foods. This is for a paper that is due in two days, so you need articles that you can read right away. Display a full text article on the screen.

3b) Print the article

3c) Now go back to your list of hits (articles).

4) You want to create a list of databases that you can come back to regularly - your favorites. Go ahead and create a list and include PsycINFO, Expanded Academic ASAP and Academic Search Premier.

5) The library owns a thesis about the mechanics of throwing a baseball by an author named Collins. Locate it.

6a) I'm looking for articles about a book called "Bluest Eye" by Toni Morrison. Choose one database from your results and display the list of hits. Don't click on any one result. You don't need to display individual articles right now.

$6 b)$ Return to the list of databases that were displayed when you did your search.

7) You want to find information on physical therapy. Select one or more Categories that covers physical therapy.

Upon the subjects' completion of the seven tasks, researchers asked them three reaction questions. The first two questions were open-ended and sought subjects' general impressions of the software. The third question targeted two components of 
the system (a link to an A-Z list of databases and an advanced search function) that researchers had anticipated subjects might overlook.

Researchers advertised for subjects on real and virtual bulletin boards, offering a small stipend for participation. Care was taken to ensure that subjects represented the library's various user populations. The four subjects were:

- $\quad$ one member of the college staff; limited searching experience;

- $\quad$ one adult learner; limited searching experience;

- one library student employee, a lower-division undergraduate; strong searching skills;

- one upper-division undergraduate; moderate searching skills.

Administering the test was simple and inexpensive. A video camera, borrowed from the college's media services department, was set up behind each subject so it could record the subject's typing motions as well as the images and words on the computer monitor. Subjects' verbalized thoughts were captured on the audio track.

When the subjects arrived, they were asked to read and sign a release, which had been approved by the college's Institutional Review Board, to show that they agreed to be part of the experiment and that their anonymity would be protected. Then the researcher explained what they were expected to do. The instructions they were given stated:

"We will ask you to do something such as looking for a journal article on 'Articles Plus.' You will proceed to do this but as you work and even before you begin typing, we want you to speak out loud everything that goes through your head. Essentially, 
you will be describing your actions. Do not worry that it may sound silly to us; it won't. Even expressions such as 'ummm, gee, let's see" should be said out loud."

It was very important that the subjects understood that they had to speak their thoughts out loud; and that the researcher could not answer questions (which proved to be the most challenging thing for the researchers, who were reference librarians).

Subjects were handed the list of tasks, and the testing began. As the subjects worked, they were both videotaped and observed by a researcher, who took notes. Subjects took between thirty minutes and an hour and fifteen minutes to complete the seven tasks. After the testing was completed and the subjects were compensated and thanked, the real work--transcribing and analyzing the test results--began.

\title{
Analysis and coding of results
}

The researchers viewed the videotapes and compiled a close transcription. Upon analysis of the notes and transcripts, they were able to group subjects' activities into ten broad categories (see fig. 3).

\section{Figure 3 - Categories identified through protocol analysis}

\author{
1. Formulating a search \\ 2. Category/database selection \\ 3. Navigation and labels \\ 4. Searching/Dealing with search results \\ 5. Revising searches \\ 6. Personalization features \\ 7. Printing \\ 8. General information literacy considerations \\ 9. Issues re: library catalogs/Searching for books \\ 10. General reaction to product
}


The researchers assigned a different color to each category and went through the transcripts, using different colored highlighters to code the data. This coding made it easy to pull together all of the problems that users experienced in each category. The researchers were then able to draw meaningful conclusions based on the aggregated experiences of all users, which would lead to concrete recommendations for how to improve the system.

\section{Findings}

This study's findings closely mirror those described elsewhere in the literature (see Literature Review). Users did, in fact, enter keyword-based search strings consistent with a Google search model. Browser-based navigation tools, especially the back button, were favored over navigation built into the ENCompass interface. Many of the same areas of confusion recounted in other studies were encountered by our users, as well. These include:

- Sequence of search actions. The implementation of ENCompass being tested required users to select one or more subject areas or databases to search before submitting their query. Failing to do so resulted in an error message instructing the user to "choose a collection." One subject in particular consistently forgot to choose a collection before clicking the search button, finally complaining, "This is not helping me!"

- Uncertainty about what types of sources were being searched (books, articles, websites, etc.). When instructed to find a specific format of information (article, book, thesis), subjects sometimes had difficulty choosing an appropriate source to 
search (database, library catalog, etc.). Some subjects were also unable to interpret results, mistaking article abstracts for book summaries.

- Lack of available search limits. Like many federated search tools, ENCompass does not allow users to limit their search to full-text documents. One of the test questions required subjects to find and display a full-text article. Subjects actively sought a means of limiting to full text; one subject asked aloud, "Is there something that allows you to limit?" and even tried to search the (non-existent) help files. Another subject opened up many records from their results set, hoping to come upon a full-text document and finally commenting, "I could find this faster searching on the regular Internet."

- Search response time. Federated searching takes longer than normal searching because multiple databases are being queried. All of the subjects commented on the slow response time of the system, but at least one subject was comforted by the animated "search in progress" bar: "I like programs that let me know they're doing something."

The out-of-the-box version of ENCompass included several design flaws that made it almost impossible for users to complete basic tasks. For example, if a user typed a search or selected one or more categories to search, and then explored any aspect of the system (viewed a description of a database, for example), all information they had entered would be lost. During testing, one subject consistently viewed database descriptions to help her select the most appropriate resource. Over and over, she displayed a description, only to lose everything she had typed when she returned to her search. Finally, she gave up, commenting that she no longer wished to 
chance losing her work. Another puzzling aspect of the system was that several very commonly used search words—Boolean operators, prepositions—-turned out to be stopwords whose inclusion in a search string would cause a search to fail. Subjects could not understand why such seemingly innocuous searches as "fitness and children" would return an error message.

A third type of problem that the researchers noted dealt with jargon or technical language. The issue of library jargon, discussed at length by Naismith and Stein (1989), is by no means a recent problem. Federated search and its associated vocabulary do throw users some new curveballs in this regard, however. Various terms used to describe groupings of resources--"collections," "categories," "repositories"--caused confusion among subjects in this study, with one lamenting that the term "collection" was "not defined anywhere." Faced with the option to "Search Native Interface," one user said, "I don't know what that is." Faced with the option to "view objects," a term that was used for article results, another subject expressed confusion: "I'm not sure if that tells me anything about it." Upon encountering the term "XML," one subject said, "View XML... riiiiight."

Protocol analysis has been noted for its ability to reveal unexpected problems or user preferences in a system. This was certainly the case in this study. Aspects of the ENCompass interface that researchers had guessed users would largely ignore turned out to be very popular with users.

One researcher had predicted before the study began that a drop-down Quick Navigation menu would be ignored by users, and actually lobbied for its removal. 
During testing, it turned out to be one of the most consistently used features by all four subjects. This observation led the researchers to recommend adding functionality to the Quick Navigation menu by incorporating more choices into it.

Even more surprising was the popularity of a small button at the bottom of the page labeled "View XML." Users would click this button multiple times, despite being presented with a screenful of unintelligible code every time they did. It seemed to be the button that users would turn to when all else failed (See Figure 4). The placement of the "View XML" button, along with the fact that it looked like a button (most of the other navigation elements were text links), proved an irresistible combination for users. This quickly led to the conclusion that the library needed to create better, more meaningful navigation elements and place them where users were likely to look for them.

\section{Figure 4. View XML examples from notes and transcripts.}

- Went right to "View XML"! But quickly closed window.

- moused over 'View XML' button but didn't click it

- afterward: Found the "View XML" button a little confusing—-kept wanting to click it to perform a search.

- Tried View XML and actually read through quite a bit of it, then closed it.

- Did View XML again.

- Clicked View XML (mistake).

- moused over (but didn't click) View XML

- afterward: 'View XML' button was "intimidating, and not helpful."

- "View XML...riiiiight."

- Did View XML. "It won’t search." Kept going back. Did "View XML" again. Twice!

- Picked another record—not full text. View XML. Back to list. Search. Lots of hits. View XML. Application error. 


\section{Recommendations}

The protocol analysis of ENCompass led to the proposal of a number of changes to the product's interface. As a small college library with a lack of programming expertise among its staff, Babson Library was not in a position to implement all of the desired interface changes. Nor were all of the changes possible to implement in the current version of the software. As a result, recommendations were divided into three groups:

(1) Changes that could be made immediately, by Babson Library staff.

(2) Changes that were beyond our staff's technical ability, but which we might be able to implement with the help of others (e.g., other libraries who had implemented the product; Endeavor Technical Support).

(3) Changes which were not possible in the current software release, but which would be submitted to Endeavor as enhancement requests.

Several changes that addressed problems discussed in this article could be made immediately, by Babson Library staff. The wording of error messages was changed to make them more meaningful: for example, the prompt "Please select a collection" was changed to "Please select one or more categories, below." Certain functions were renamed for clarity, e.g. "View Resource" became "View Full Text." It was recommended that the "Search Native Interface" button that appears in the results be customized so that the name of the actual database (e.g., PsycINFO) would display. As mentioned above, more navigation options were added to the Quick Navigation 
drop-down menu, which was a popular feature with all four subjects. The "View XML" button was removed.

Some changes required the library to seek help from other libraries that had implemented ENCompass and had greater programming skills. One library was able to share with us a script they had written that would strip stopwords out of userentered queries. This eliminated the problem whereby commonly used search terms, like Boolean operators, would cause a search to fail. This change alone improved the usability of the product immensely.

Finally, certain recommendations were submitted to the vendor, Endeavor, as enhancement requests. For example, the issue where users lost all information they had entered every time they viewed a database description was something that could only be addressed in future product releases.

\section{Outcomes and Lessons Learned}

Despite the various difficulties that they encountered during testing, subjects' reactions to the concept of federated search were unanimously positive, with all four commenting that they looked forward to using such a tool in their research. The staff of Babson Library was pleased to be able to incorporate the findings from this study into their version of ENCompass, Articles Plus, before launching it in the fall of 2003. Figure 5 shows an example of the reconfigured Articles Plus single record view screen. 


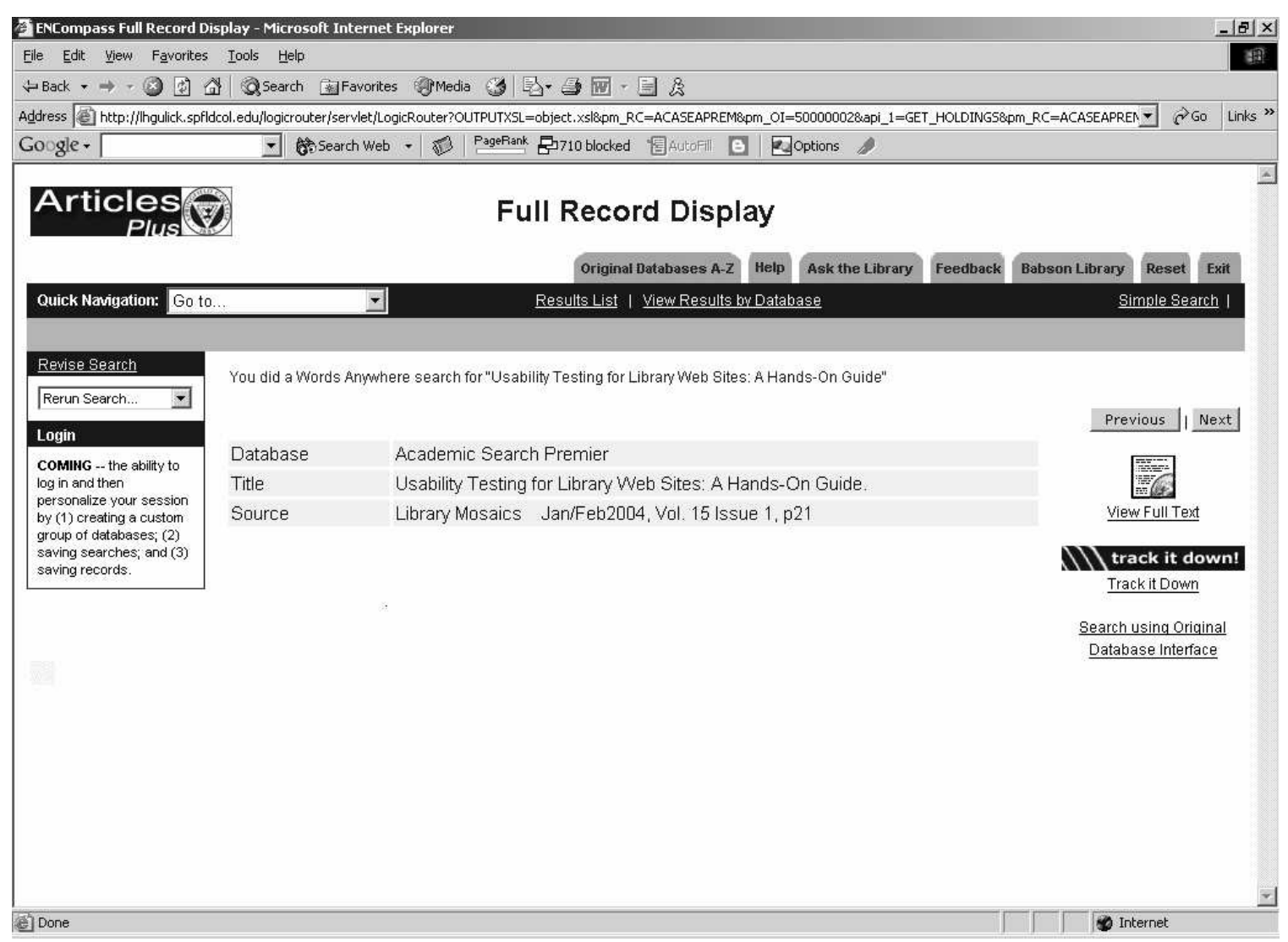

(Figure 5: Articles Plus single record screen following redesign)

Lessons learned by the researchers during this study include:

\section{- Video cameras are not the best method of recording activity on a computer}

screen. The researchers would turn to a screen-capture tool, such as Camtasia or

Captivate, for this purpose in the future. Videotaping the testing is still a good

idea, however, in order to capture subjects' verbalizations and non-verbal cues.

- An exact transcript is preferable to rough notes. Compiling a loose, rather

than an exact, transcription of the testing tapes can be a time-saver, but

researchers may later regret not having access to subjects' exact words. It is up to

each library to decide whether an exact transcription is worthwhile. 
- Usability testing is an iterative process. This mantra, much repeated in the literature (e.g., Brown 2003, Nielsen 2000) is absolutely true. As the needs of users and the possibilities inherent in the technology change, so must libraries' services. A year after this study, Articles Plus was given a more radical makeover featuring a greatly simplified, Google-like interface. These subsequent changes, which eliminated many of the problems that remained after the first round of improvements, came about as a result of further usability testing, product enhancements, and stronger programming skills among staff.

- Finally, as libraries gain more control over the look and feel of the products they purchase or license, it becomes increasingly important for them to hire or develop staff with coding or programming skills. Babson Library relied heavily on one staff member who was self-taught in XML and XSL to make the changes recommended in this study. Greater staff expertise in this area would have allowed the library to make more changes, and would also have given the library staff a better understanding of what changes were possible. Even as the fairly modest changes recommended in this report were being enacted, other, larger ENCompass customers with coders on staff, like the University of Rochester and the University of Kansas (both of whom graciously shared their expertise with Babson Library), were customizing their interfaces in ways that “code-poor" libraries could only dream of.

Federated search presents libraries with a great opportunity to draw in users by offering a way for people to locate trusted information with one, simple search. If the vendor falls short in the version it provides, then it falls to libraries to ensure that as 
few usability barriers as possible stand between the searcher and the information sought. This can no longer be done without the requisite technology skills.

\section{Conclusion}

Federated searching, by its very nature, can present usability challenges for searchers who are accustomed to tools that search a discrete set of information and offer a full suite of search limits and options. These challenges can be compounded by the federated search software's interface design. Usability testing via protocol analysis can give libraries valuable insights as to how users interact with the fairly young technology of federated searching. Conclusions drawn from observing actual user behavior will inform a library's decisions about how best to customize the federated search interface. Babson Library's protocol analysis of ENCompass resulted in the recommendation of a number of interface changes that produced a more usable—and useful—product for the library's users.

\section{REFERENCES}

Brown, Stephanie Willen. 2002. Test, edit, repeat: Steps to improve your web site. Computers in Libraries 22 (10): 14-16.

Cervone, Frank. 2005. What we've learned from doing usability testing on OpenURL resolvers and federated search engines. Computers in Libraries 25 (9): 10-14.

Ericsson, K. Anders, and Herbert A. Simon. 1980. Verbal reports as data. Psychological Review 87 (3): 215-251. 
Ericsson, K. Anders, and Herbert Alexander Simon. 1993. Protocol analysis: Verbal reports as data. Rev. ed. Cambridge, Mass.: MIT Press.

Gilhooly, K., and C. Green. 1996. Protocol analysis: Theoretical background. In Handbook of qualitative research methods for psychology and the social sciences, ed. Richardson, J. T. E., 43-74. Leicester: BPS Books.

Letnikova, Galina. 2003. Usability testing of academic library web sites: A selective annotated bibliography. Internet Reference Services Quarterly 8 (4): 53-68. doi:10.1300/J136v08n04_04.

Mack, Thura, Maribeth Manoff, and Tamara J. Miller. 2004. Designing for experts: How scholars approach an academic library web site. Information Technology and Libraries 23 (1): 16-22.

Naismith, Rachael, and Joan Stein. 1989. Library jargon: Student comprehension of technical language used by librarians. College \& Research Libraries 50 (9): 543-52.

Nielsen, Jakob. 2000. Why you only need to test with five users. Alertbox (March 19, 2000), http://www.useit.com/alertbox/20000319.html (accessed February 20, 2006).

Novotny, Eric. 2004. I don't think I click: A protocol analysis study of use of a library online catalog in the Internet age. College \& Research Libraries 65 (6): (11).

Pace, Andrew K. 2003. The usability toolbox. Computers in Libraries 23 (1): 50. 
Ponsford, Bennett Claire. 2005. Usability testing of MetaLib 3.12. Presented at SFX/Metalib Users Group Meeting, June 9-10, 2005, College Park, Maryland. http://smugnet.org/2005na/presentations/PonsfordUsability-Testing.ppt (accessed April 23, 2006).

Schmidt, Kari, and Laura Wrubel. 2005. Metasearch usability: Student reactions. Presented at SFX/Metalib Users Group Meeting, June 9, 2005, College Park, Maryland. http://smugnet.org/2005na/presentations/SMUG-schmidt-wrubel.ppt (accessed April 23, 2006).

Tallent, Ed. 2004. Metasearching in Boston College Libraries - a case study of user reactions. New Library World 105 (1/2): 69-75 (accessed April 16, 2006).

Van House, Nancy A., Mark H. Butler, Virginia Ogle, and Lisa Schiff. 1996. Usercentered iterative design for digital libraries: The Cypress experience. D-Lib Magazine (February 1996), http://www.dlib.org/dlib/february96/02vanhouse.html (accessed February 19, 2006).

Wiedenbeck, Susan, Robin Lampert, and Jean Scholtz. 1989. Using protocol analysis to study the user interface. Bulletin of the American Society for Information Science 15 (6): $25-6$. 